\title{
Effect of Substrates and Thermal Treatments on Metalorganic Chemical Vapor Deposition-Grown $\mathrm{Sb}_{2} \mathrm{Te}_{3}$ Thin Films
}

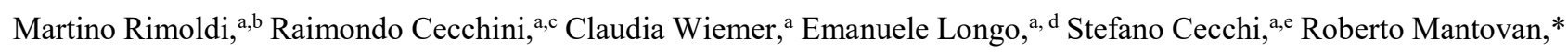

a Massimo Longo* a,f

${ }^{a}$ Institute for Microelectronics and Microsystems, CNR-IMM Unit of Agrate Brianza, via C. Olivetti 2, 20864, Agrate Brianza, Italy.

${ }^{\mathrm{b}}$ Currently at: CERN, European Organization for Nuclear Research, 1211, Geneva 23, Switzerland.

${ }^{c}$ Currently at: Institute for Microelectronics and Microsystems, CNR-IMM Unit of Bologna, via P. Gobetti 101, 40129, Bologna, Italy.

${ }^{\mathrm{d}}$ University of Milano-Bicocca, Department of Material Science, Via R. Cozzi 55, 20126, Milan, Italy.

e Currently at: Paul-Drude-Institut für Festkörperelektronik, Leibniz-Institut im Forschungsverbund Berlin e.V., Hausvogteiplatz 5-7, 10117 Berlin, Germany.

${ }^{\mathrm{f}}$ Currently at: Institute for Microelectronics and Microsystems, CNR-IMM Unit of Rome, via Fosso del cavaliere, 100 , 00133 Rome, Italy.

* roberto.mantovan@mdm.imm.cnr.it (R. Mantovan)

* massimo.longo@artov.imm.cnr.it (M. Longo)

\section{Supporting Information}

Table of Contents

Page

1. SEM Images

2. AFM Images

3. XRR Measurements

4. XRD Measurements

5. References 


\section{SEM Images}
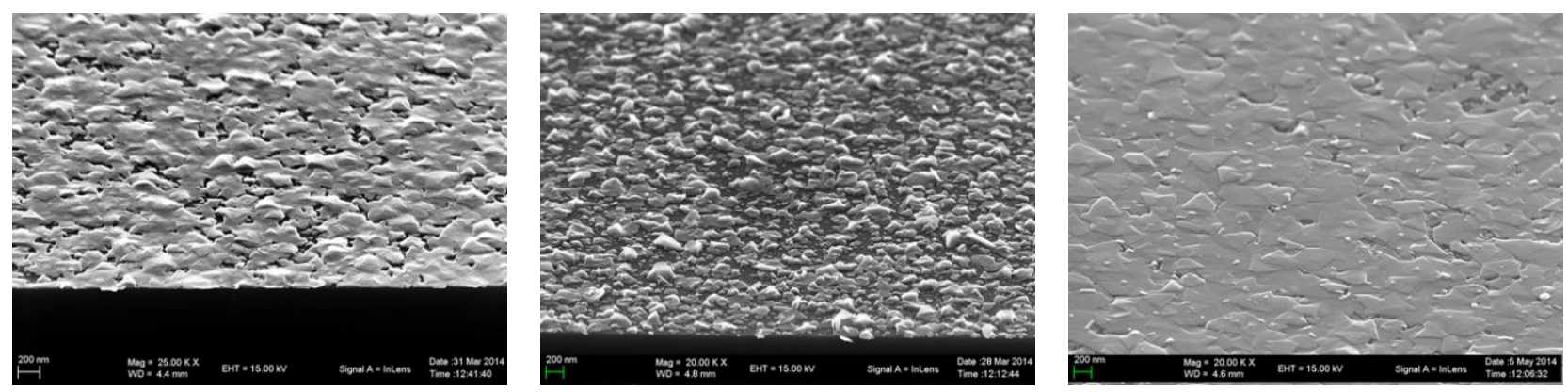

Figure S1. Tilted SEM images of $\mathrm{Sb}_{2} \mathrm{Te}_{3} / \mathrm{Si}(111)$ grown by MOCVD at (from left to right) $100^{\circ} \mathrm{C}, 150^{\circ} \mathrm{C}$, and $250^{\circ} \mathrm{C}$. 


\section{AFM Images}

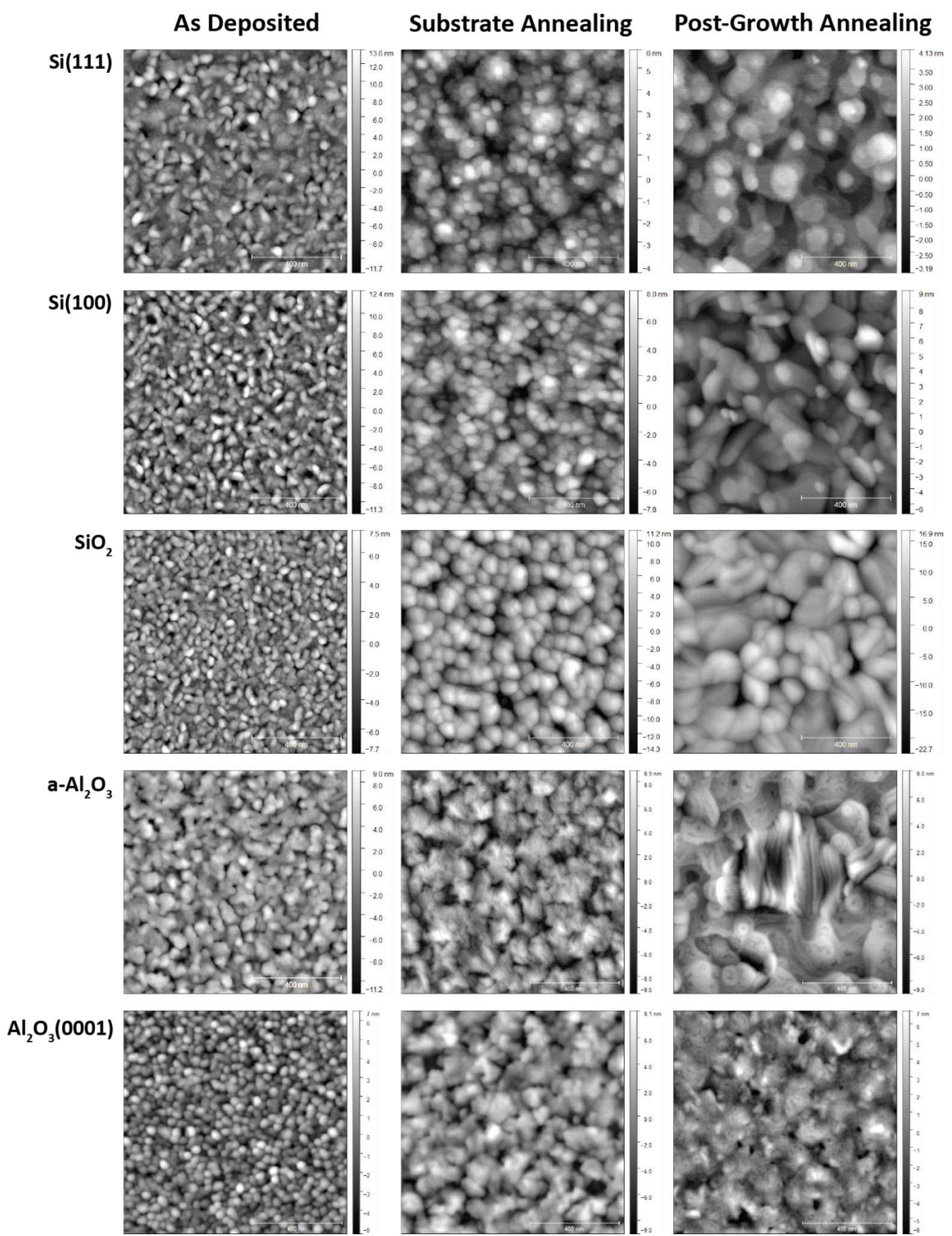

Figure S2. Planar view AFM images of (left) $\mathrm{Sb}_{2} \mathrm{Te}_{3}-$ as-deposited, (middle) $\mathrm{Sb}_{2} \mathrm{Te}_{3}$ - substrate annealing, and (right) $\mathrm{Sb}_{2} \mathrm{Te}_{3}$ - post-growth annealing on (from top to bottom) $\mathrm{Si}(111), \mathrm{Si}(100), \mathrm{SiO}_{2}, \mathrm{a}_{-} \mathrm{Al}_{2} \mathrm{O}_{3}$, and $\mathrm{Al}_{2} \mathrm{O}_{3}(0001)$. $\mathrm{Si}(111)$ is reported for comparison purpose. ${ }^{1}$ 


\section{XRR Measurements}
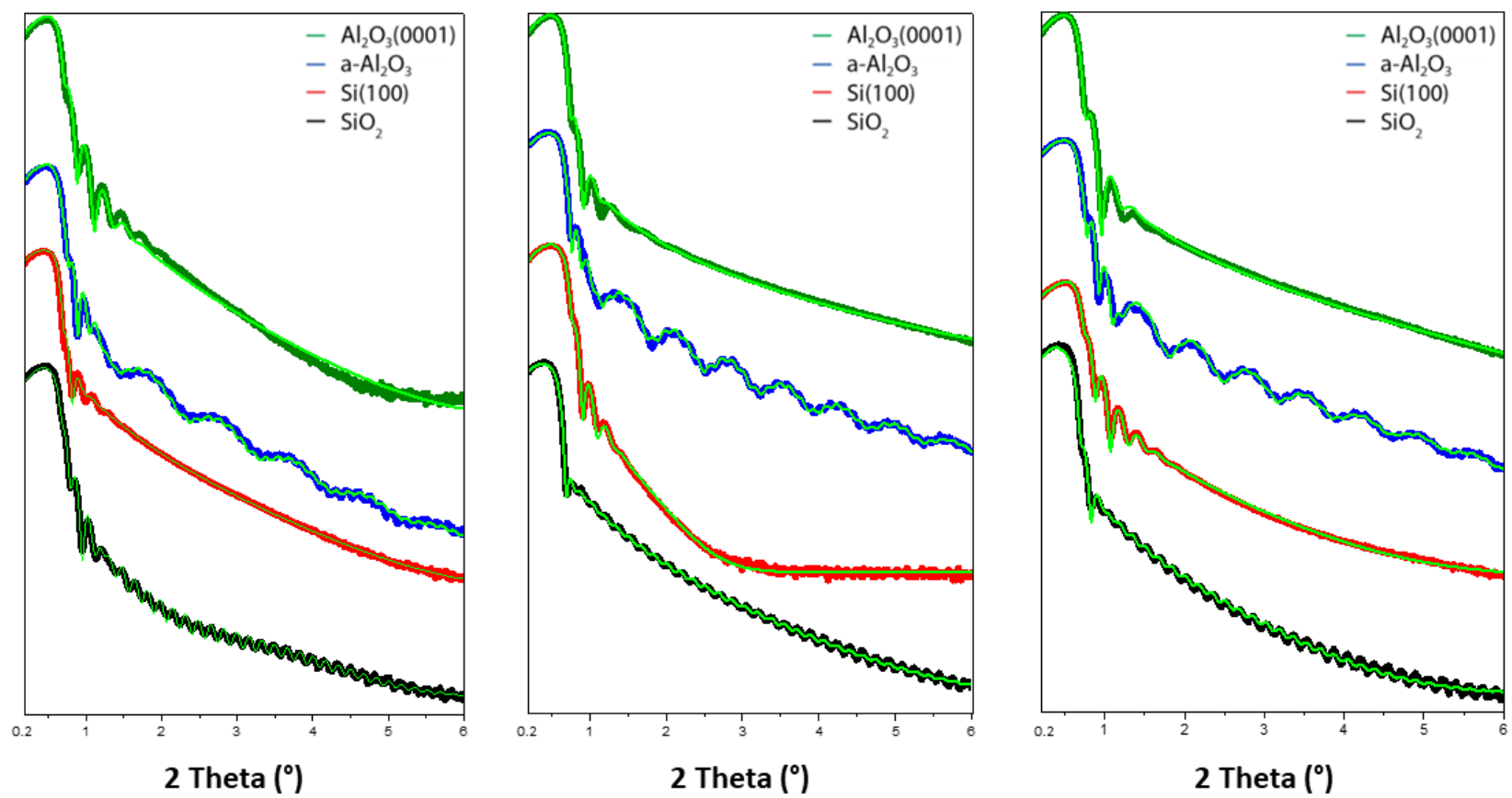

Figure S3. XRR measurements of (left) as-deposited, (middle) substrate annealing, and (right) post-growth annealing $\mathrm{Sb}_{2} \mathrm{Te}_{3}$ on (green) $\mathrm{Al}_{2} \mathrm{O}_{3}\left(0001\right.$ ), (blue) a- $\mathrm{Al}_{2} \mathrm{O}_{3}$, (red) $\mathrm{Si}(100)$, and (black) $\mathrm{SiO}_{2}$.

\section{XRD Measurements}
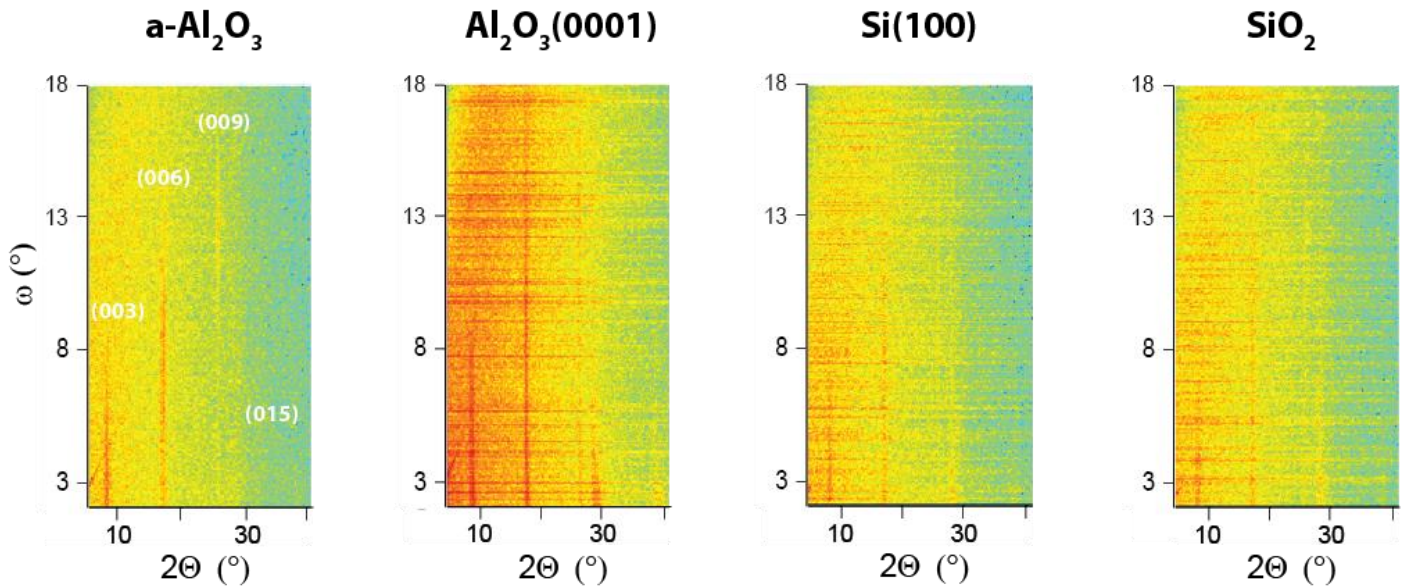

Figure S4. XRD maps in Bragg-Brentano geometry of $\mathrm{Sb}_{2} \mathrm{Te}_{3}$ - as-deposited on a- $\mathrm{Al}_{2} \mathrm{O}_{3}, \mathrm{Al}_{2} \mathrm{O}_{3}(0001), \mathrm{Si}(100)$, and $\mathrm{SiO}$. 

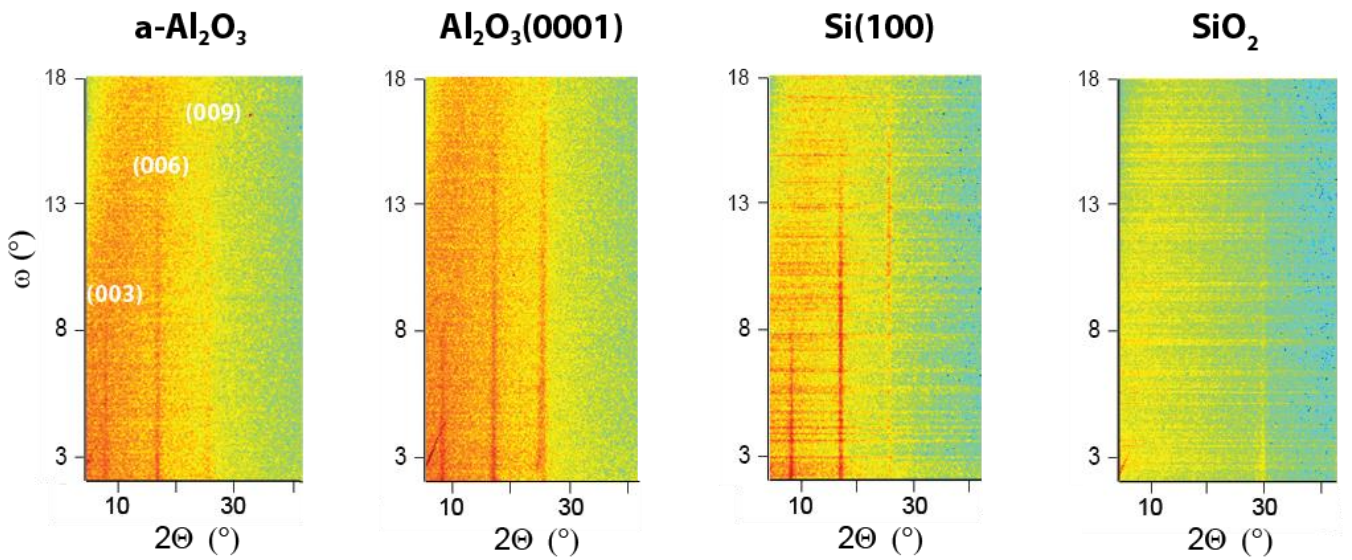

Figure S5. XRD maps in Bragg-Brentano geometry of $\mathrm{Sb}_{2} \mathrm{Te}_{3}-$ substrate annealing on a- $\mathrm{Al}_{2} \mathrm{O}_{3}, \mathrm{Al}_{2} \mathrm{O}_{3}(0001), \mathrm{Si}(100)$, and $\mathrm{SiO}_{2}$.
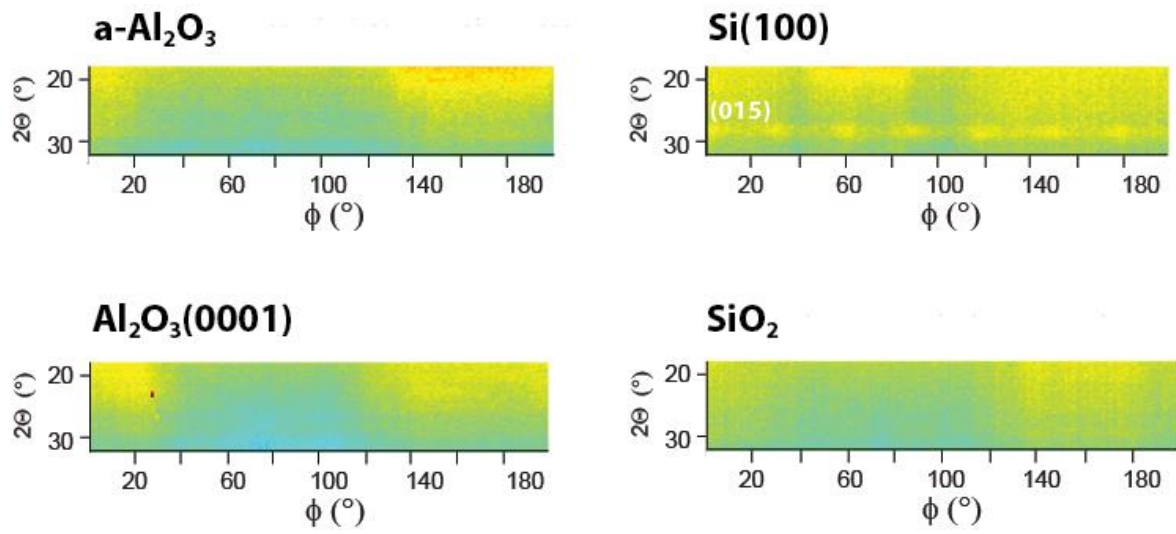

Figure S6. XRD $\phi$ angle scan measurement of $\mathrm{Sb}_{2} \mathrm{Te}_{3}-$ substrate annealing on a- $\mathrm{Al}_{2} \mathrm{O}_{3}, \mathrm{Al}_{2} \mathrm{O}_{3}(0001), \mathrm{Si}(100)$, and $\mathrm{SiO}$.
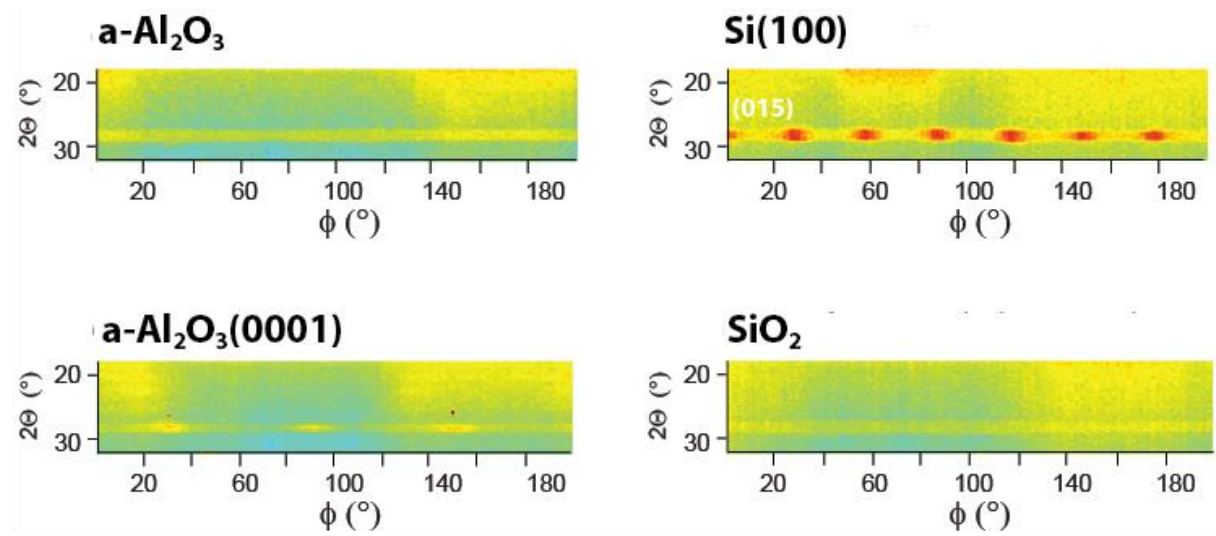

Figure S7. XRD $\phi$ angle scan measurement of $\mathrm{Sb}_{2} \mathrm{Te}_{3}$ - post-growth annealing on a- $\mathrm{Al}_{2} \mathrm{O}_{3}, \mathrm{Al}_{2} \mathrm{O}_{3}(0001), \mathrm{Si}(100)$, and $\mathrm{SiO}_{2}$.

\section{References}

1. M. Rimoldi, R. Cecchini, C. Wiemer, A. Lamperti, E. Longo, L. Nasi, L. Lazzarini, R. Mantovan and M. Longo, RSC Adv., 2020, 10, 19936-19942. 